University of Nebraska - Lincoln

DigitalCommons@University of Nebraska - Lincoln

\title{
Fusarium genomic resources: Tools to limit crop diseases and mycotoxin contamination
}

Daren W. Brown

USDA-ARS, daren.brown@ars.usda.gov

Robert A. E. Butchko

USDA-ARS

Robert H. Proctor

USDA-ARS

Follow this and additional works at: https://digitalcommons.unl.edu/usdaarsfacpub

Brown, Daren W.; Butchko, Robert A. E.; and Proctor, Robert H., "Fusarium genomic resources: Tools to limit crop diseases and mycotoxin contamination" (2006). Publications from USDA-ARS / UNL Faculty. 1580.

https://digitalcommons.unl.edu/usdaarsfacpub/1580

This Article is brought to you for free and open access by the U.S. Department of Agriculture: Agricultural Research Service, Lincoln, Nebraska at DigitalCommons@University of Nebraska - Lincoln. It has been accepted for inclusion in Publications from USDA-ARS / UNL Faculty by an authorized administrator of DigitalCommons@University of Nebraska - Lincoln. 


\title{
Fusarium genomic resources: Tools to limit crop diseases and mycotoxin contamination
}

\author{
Daren W. Brown, Robert A. E. Butchko \& Robert H. Proctor \\ Mycotoxin Research Group, USDA/ARS, $1815 \mathrm{~N}$ University St, Peoria, IL, 61604, USA
}

\begin{abstract}
It has been almost 10 years since Joan Bennett suggested that fungal biologists create a "wish list" for fungal genome sequences (Bennett JW. White paper: Genomics for filamentous fungi. Fungal Genet Biol 1997; 21: 3-7). The availability of over 200 review papers concerning fungal genomics is a reflection of significant progress with a diversity of fungal species. Although much progress has been made, the use of genomic data to study mycotoxin synthesis and function, pathogenesis and other aspects of fungal biology is in its infancy. Here, we briefly present the status of publicly available genomic resources for Fusarium, a genus of important plant pathogenic and mycotoxin-producing fungi of worldwide concern. Preliminary examination of microarray data collected from $F$. verticillioides liquid cultures provides evidence of widespread differential gene expression over time.
\end{abstract}

Key words: ESTs, Fusarium genomics, F. verticillioides, fumonisin regulation, microarray, zinc finger

Abbreviations: NCBI - National Center for Biotechnology Information; TIGR - The Institute for Genomics Research; DOE JGI - U.S. Department of Energy Joint Genome Institute

\section{Introduction}

The genus Fusarium includes numerous plant pathogens that cause destructive diseases on some of the world's most agriculturally important plant species, including corn, wheat, potato, cassava, palm, banana, pine, and numerous vegetables. Some pathogenic Fusarium species also produce mycotoxins that pose health risks to humans and animals and that markedly reduce the value of the crops in which they occur. Fusarium mycotoxins span a structurally diverse array of compounds and are produced in infected plant tissue. They become a safety and economic concern when they accumulate in food or feeds to levels toxic to ingesting organisms. Fusarium is distributed worldwide and is highly abundant in some environments. In some agricultural fields, there are as many as 100,000 Fusarium propagules per gram of soil [1]. The wide distribution of Fusarium is likely due to their ability to colonize diverse plants and soils and their ability to disperse widely [2]. Plant infections by Fusarium can occur at all developmental stages, from developing or germinating seeds to mature vegetative tissues, depending on the host plant and Fusarium species involved. Root, stalk and ear rots, blights and wilts are a few of the diseases incited by the fungus.

Fusarium graminearum and $F$. verticillioides are two of the most economically important Fusarium species in many regions of the world. F. graminearum is a pathogen of wheat, corn and barley and, over the past 15 years, has caused multibillion dollar losses through reduced yields and contamination of grain with the mycotoxins deoxynivalenol (DON) and/or zearalenone. DON inhibits protein synthesis, while zearalenone has estrogenic activity and has been associated with reproductive disorders in livestock [reviewed in 3]. The synthesis of DON and zearalenone exemplifies two different biosynthetic origins for a substantial percentage of fungal secondary metabolites. DON 
is a sesquiterpene (a member of the trichothecene family of compounds) and is synthesized by the cyclization of the isoprenoid intermediate farnesyl pyrophosphate by a trichodiene synthase. Trichodiene, the first intermediate in the pathway, then undergoes up to 14 oxygenations, isomerations, cyclizations and estiferications to form the final product [4]. Zearalenone is a polyketide and is synthesized by the condensation of acetyl units by a polyketide synthase (PKS). In contrast to many other metabolites derived from polyketides, the synthesis of zearalenone requires two PKSs and does not appear to require any post-PKS modifying reactions [5].

$F$. verticillioides, also a pathogen of corn, can contaminate kernels with a family of closely related mycotoxins known as fumonisins. These toxins disrupt sphingolipid metabolism through the inhibition of the enzyme ceramide synthase. Fumonisins are polyketides and, in contrast to zearalenone, the PKS generated intermediate undergoes up to nine oxygenation, esterification, reduction and dehydration reactions to form the final product $[6,7]$.

Much research over the past 20 years has focused on understanding the biochemical and regulatory pathways required for Fusarium mycotoxin biosynthesis. The overall goal of this research has been to generate novel methods to limit fungal disease on wheat and/or corn and to limit mycotoxin contamination of human food and animal feeds. Although significant advances have been made towards understanding toxin biosynthesis as well as basic fungal biology, progress towards developing new methods to limit disease is hampered by the slow process of identifying target genes for further study. A number of years ago, the fungal research community recognized that genomic resources could lead to dramatic advances in understanding mycotoxin biosynthesis, pathogenesis of fungal-plant interactions and the development of new fungal control strategies [8]. This review will discuss the current status of genomic resources available principally for $F$. graminearum and $F$. verticillioides (Table 1).

\section{$F$. graminearum genomic resources}

F. graminearum (teleomorph Gibberella zeae) has an estimated genome size of $\sim 36 \mathrm{Mb}$ that span four chromosomes. At present, the genomic resources available include two genetic maps, genome sequence, and an expressed sequence tag (EST) library [9, 10 and www.broad.mit.edu/annotation/ genome/fusarium_graminearum/Home.html]. The first genetic map is based on molecular markers and a cross between two unrelated parent strains derived from a Japanese strain (R-5470) and a US strain (GZ3639). It consists of nine linkage group and spans $1300 \mathrm{cM}$ or map units with an average interval of 2.8 map units between loci [11]. Three of the nine linkage groups exhibited high levels of segregation distortion [11]. This may be explained by recent DNA-based phylogenetic evidence suggesting the parents of the cross, GZ3639 and $\mathrm{R} 5470$, were different species; $F$. graminearum (previously $F$. graminearum lineage 7 ) and $F$. asiaticum (previously $F$. graminearum lineage 6), respectively [12]. Fifty-two of the markers examined in the cross were later sequenced and were found to anchor $99 \%$ of the genome sequence of $F$. graminearum strain $\mathrm{PH}-1$ (www.broad.mit. edu/annotation/genome/fusarium_graminearum/ markers.html). The second map is based on a cross between parent strains derived from two $F$. graminearum strains (PH-1 and 00-676) and consists of 11 linkage groups which cover $1154 \mathrm{cM}$. Of the markers, 162 were sequenced and found to anchor $99.6 \%$ of the genome sequence (see below). All linkage groups and anchored supercontigs could be assembled into the four chromosomes, leaving 11 smaller supercontigs (representing less than $75 \mathrm{~kb}$ ) not anchored. Segregation distortion was observed for 17 markers at four genomic locations.

The publicly available $F$. graminearum Expressed Sequence Tags (ESTs) were produced from three different cDNA libraries [10]. A total of 7996 ESTs were generated and these corresponded to 2,110 unique sequences. Two libraries were created from carbon- or nitrogen-limited media and the third was created from cultures of maturing perithecia. The libraries included sequences corresponding to numerous genes with homology to known fungal genes required for virulence or pathogenicity. The observation that less than $22 \%$ of the ESTs in the nitrogen-limited library shared significant similarity $\left(E<1 \times 10^{-5}\right)$ to a comparable library from Magnaporthe grisea suggests that the majority of $F$. graminearum ESTs do not have M. grisea homologues [10]. 
Table 1. Web resources to Fusarium genomic resources

\begin{tabular}{|c|c|c|c|c|}
\hline Fusarium & Sequence & Organization & \multicolumn{2}{|c|}{ Reference Web site } \\
\hline \multicolumn{2}{|c|}{ F. verticillioides Genetic Map } & Kansas State Univ. & [9] & $\mathrm{NA}^{\mathrm{a}}$ \\
\hline \multicolumn{3}{|c|}{ F. verticillioides $\mathrm{ESTs}(87,000+) \mathrm{TIGR}$} & [14] & www.tigr.org/tigr-scripts/tgi/T_index.cgi?species =f_verticill \\
\hline \multicolumn{2}{|c|}{ F. verticillioides Genomic ${ }^{\mathrm{b}}$} & $\begin{array}{l}\text { Syngenta and Broad } \\
\text { Institute }\end{array}$ & $\mathrm{Na}$ & $\begin{array}{l}\text { www.broad.mit.edu/annotation/genome/fusarium_verticillioides/ } \\
\text { Home.html }\end{array}$ \\
\hline F. graminearun & $n$ Genetic Maps & $\begin{array}{l}\text { Kansas State Univ. } \\
\text { and Broad Institute }\end{array}$ & [11] & $\begin{array}{l}\text { www.broad.mit.edu/annotation/genome/fusarium_graminearum/ } \\
\text { markers.html }\end{array}$ \\
\hline F. graminearun & $n$ Genomic & Broad Institute & NA & $\begin{array}{l}\text { www.broad.mit.edu/annotation/genome/fusarium_graminearum/ } \\
\text { Home.html }\end{array}$ \\
\hline F. graminear & $n$ ESTs $(10,000+$ & Michi & .110] & (1) \\
\hline F. oxysporum & Genomic $^{c}$ & Broad Institute & NA & $\begin{array}{l}\text { www.broad.mit.edu/annotation/genome/fusarium_graminearum/ } \\
\text { Home.html }\end{array}$ \\
\hline F. solani & Genomic $^{c}$ & DOE JGI ${ }^{\mathrm{d}}$ & $\mathrm{Na}$ & www.microbialgenome.org/organisms.shtml \\
\hline
\end{tabular}

${ }^{\mathrm{a}} \mathrm{NA}=$ Not applicable.

${ }^{\mathrm{b}} 4 \times$ coverage of $F$. verticillioides was made available from Syngenta and curated by the Broad Institute and funding for additional genomic sequence has been approved.

${ }^{\mathrm{c}}$ In progress.

${ }^{\mathrm{d}}$ DOE JGI $=$ Department of Energy Joint Genome Institute.

The $F$. graminearum whole genome sequencing project of strain $\mathrm{PH}-1$ was initiated by state university (Frances Trail and Jin-Rong $\mathrm{Zu}$ ) and USDA (Corby Kistler) researchers and funded by the USDA/NSF Microbial Genome Sequencing Project in collaboration with the Broad Institute at the Massachusetts Institute of Technology. The entire project, sequence methodology and summary has been posted on the Broad Institute website (www.broad.mit.edu/annotation/genome/ fusarium_verticillioides/Home.html). The sequence was generated by end sequencing numerous plasmids, fosmids and BAC clones to produce $10 \times$ sequence coverage of the genome. The sequences were assembled into 511 contigs (longer than $2 \mathrm{~kb}$ ) with a total combined contig length of over 36 million bp (GenBank accession AACM00000000). The information from paired reads (sequence reads from the same clone) lying on different contigs was used to order and link different contigs into a total of 28 supercontigs or scaffolds. The availability of this genomic resource will allow the comparative analysis of $F$. graminearum with other maize and wheat pathogens.

\section{$F$. verticillioides genomic resources}

F. verticillioides (teleomorph Gibberella moniliformis) has an estimated genome size of $\sim 46 \mathrm{Mb}$ that span 12 chromosomes. At present, the genomic resources available include genetic maps, genome sequence and an EST library. The most extensive genetic map was generated from 636 biochemical, molecular and morphological markers and a mapping population consisting of 121 meiotic progeny. The map consists of 12 linkage groups and spans 2,188 cM (map units) with an average interval of 3.9 map units between loci $[9,13]$.

A first draft of a $F$. verticillioides genome sequence was recently made available to the public and consists of a $\sim 4 \times$ whole-genome shotgun assembly (GenBank accession AAIM01000000). The sequence reads were donated to the Broad Institute by Syngenta (Basel, Switzerland) with few restrictions. Recently, Fusarium researchers and the Broad institute received funding to produce additional $F$. verticillioides genomic sequences. This additional sequence, coupled with genome sequence from $F$. graminearum and $F$. oxysporum, will be compared to generate a comprehensive Fusarium gene list. For more information, please see www.broad.mit.edu/annotation/genome/ fusarium_verticillioides/Home.html.

A majority of the publicly available $F$. verticillioides ESTs were generated by two labs [14]. The first project involved a subtraction library enriched for $F$. verticillioides transcripts expressed during fumonisin biosynthesis on maize kernels and resulted in 655 ESTs [15]. The second project produced over 87,000 ESTs from nine different cDNA libraries and correspond to 11,119 unique sequences. The different libraries were chosen in 
order to examine differential transcript expression as well as to maximize the EST variety. Four libraries were generated after growth for different lengths of time on a medium that supports fumonisin biosynthesis and five libraries were generated after growth on media containing various maize components. As was observed in F. graminearum ESTs, numerous genes were found with homology to known fungal virulence or pathogenicity factors [14]. The Institute for Genomics Research (TIGR) has integrated all publicly available $F$. verticillioides EST and gene sequence data to create a non-redundant database referred to as the $F$. verticillioides Gene Index or FvGI (www.tigr.org/tigr-scripts/tgi/T_index.cgi? species $\left.=f \_v e r t i c i l l\right)$. Release 7.0 (March, 2005) has a total of 11,126 unique sequences where each sequence represents at least one different gene. Based on a comparison to the recently released $F$. graminearum genome sequence and annotation, we estimate that the FvGI represents approximately $80 \%$ of all $F$. verticillioides genes.

\section{ESTs and whole genome sequence}

The acquisition of whole genomic sequence and/ or ESTs are important steps towards furthering our understanding of how fungi cause disease, the fungal lifecycle, and the synthesis and function of fungal secondary metabolites. A common outcome of sequencing projects is a list of all possible genes encoded by the genome. Unfortunately, the gene prediction utilities currently in use are often inaccurate and as a result cause numerous errors, including missed genes or genes with discrepancies in their structure. An example of the challenge of gene prediction is provided by comparing two efforts to predict genes from the $F$. graminearum genomic sequence data generated by the Broad Institute (www.broad.mit. edu/annotation/genome/fusarium_graminearum/ GenomeStats.html). Using the gene prediction utilities at Broad, a total of 11,640 genes were predicted. In contrast, the Munich Informatics Center for Protein Sequences (MIPS) applied their own suite of utilities to the same sequence data and predicted over 13,938 genes (mips. gsf.de/genre/proj/fusarium). Efforts to provide a more accurate gene set by comparing the
F. graminearum genome to other, closely related Fusarium genomes (see below), are in progress.

EST data coupled with genomic sequence data can significantly enhance the accuracy and precision of gene predictions. A representative example can be found in the analysis of transcripts to FUM genes located in the fumonisin biosynthetic gene cluster [14]. Complete coding sequences for each putative FUM gene were predicted based on a combination of similarity with previously characterized proteins, existing ORFs and presence or absence of intron border sequences [7]. A comparison of the FUM gene ESTs to genomic sequence corresponding to the fumonisin cluster led to the identification of new introns or intron borders in nine of the original 15 genes and enabled us to adjust the predicted protein sequences appropriately. In some cases, predicted protein changes were minimal. For example, the new predicted Fum 12p had 9 AA deleted near the amino terminus while the new Fum13p had 5 AA added. In other cases, the predicted protein changes are more extensive. For example, the new Fum8p had 51 AA changed within the middle of the protein while Fum 10p had 59 AA added to the carboxyl terminus and Fum15p had 20 AA deleted near the carboxyl terminus [14].

Analysis of genomic sequence alone can often miss genes entirely. Comparison of the $42.5 \mathrm{~kb}$ FUM cluster and flanking genomic sequence to the TIGR $F$. verticillioides EST Gene Index led to the identification of two new putative FUM genes. The first gene was designated FUM2O and was identified by a single EST (FVGBR83TH) of $680 \mathrm{bp}$ that includes a poly A track and one intron with typical border sequences [14]. FUM20 is located between FUM12 and FUM13 in the gene cluster. The second gene was designated FUM21, was identified from 16 ESTs, and encodes a putative $\mathrm{Zn}(\mathrm{II})_{2} \mathrm{Cys}_{6}$ transcriptional factor [16]. FUM21 is located adjacent to FUMI and extends the breadth of the cluster by $4 \mathrm{~kb}$. BLASTX analysis of the genomic sequence corresponding to the ESTs did not reveal significant similarity to any previously described genes (Score $\approx 30$ bits, $E \approx 1)$. In contrast, the exclusion of eight intron sequences generated a FUM21 consensus sequence that, by BLASTX analysis, shared significant similarity to numerous genes encoding $\mathrm{Zn}(\mathrm{II})_{2} \mathrm{Cys}_{6}$ transcriptional factors 
(Score $\approx 50$ bits, $E \approx 5 \times 10^{-5}$ ). Functional gene analysis is in progress and preliminary results indicates that this gene is required for fumonisin biosynthesis [16].

\section{Comparing genomic resources}

Deciphering all the functional information from genomic resources is a major challenge. Acquisition of EST or genomic sequence data is just the start of the process. Although significant insight into function can be obtained by initial comparisons to existing gene and protein databases via BLAST analysis, further experimentation is required to generate an accurate gene set. Comparative genomics, defined as the analysis and comparison of genomes from different species, is proving to be a powerful tool in the identification and characterization of genes and noncoding regions. For example, a comparative genomic analysis of three related Saccharomyces species led to the identification of hundreds of new genes, numerous annotation changes to existing ORFs, and elimination of about 500 previously annotated ORFs [17]. Equally exciting are new insights into conserved promoter sequences provided by comparative genomics. Analysis of intergenic regions in the three Saccharomyces species led to the identification of 72 well-conserved sequence motifs upstream of protein coding regions. Although many of these motifs have been previously characterized, numerous new motifs were found [17]. By examining the function of genes adjacent to conserved motifs, researchers were able to infer functions to some of the new motifs. Most of the new motifs found correlate strongly to specific functional categories [17].

Comparative genomics of closely related filamentous fungi is underway with Aspergillus and will soon be applied to Fusarium. The Aspergillus analysis has revealed a set of genes common to A. nidulans, A. oryzae and A. fumigatus as well as a much smaller set of genes unique to each [18]. In Fusarium, the genomic sequences from three related Fusaria; $F$. oxysporum, $F$. verticillioides and $F$. graminearum, will be compared to study their biology and evolution (Charlie Woloshuk, Pers. Commun.). The overall goals are to create a Fusarium gene catalogue, identify conserved noncoding elements and to characterize species-specific gene sets. More information about the Broad Institute's efforts to apply comparative sequence analysis to study Aspergillus and Fusarium cellular processes can be found at www.broad.mit.edu/ annotation/fungi/fgi/.

In 2005, the U.S. Department of Energy Joint Genome Institute (DOE JGI; www.microbialgenome.org/organisms.shtml) began sequencing the genome of a more distantly related species $F$. solani. This fungus is an important plant pathogen on numerous vegetable species. In addition, the fungus is well known for its ability to degrade a variety of complicated natural and synthetic aromatic materials and therefore may play a significant role in bioremediation projects in the future. A comparison of the more distant $F$. solani genome to the more closely related $F$. oxysporum, $F$. verticillioides and $F$. graminearum genomes will provide information about unique host specific genes (see below) and about the evolution of Fusarium genomes.

Comparative genomic analysis can also be applied to genome sequences of more distantly related organisms. For example, analysis of three protozoan pathogen genomes ranging in coding capacity from 8,100 to 12,000 genes with different life cycles and disease pathology, led to the identification of a conserved core proteome of about 6,200 genes in large clusters and numerous new unique genes that may be involved with disease in different hosts [19]. Interestingly, genes encoding elements that contribute to antigenic variation and diversity or related to immune evasion strategies, are located near telomeres which may enhance recombination frequency [19].

Recently, we compared the $F$. verticillioides EST collection against the genomic sequences of $F$. graminearum, another pathogen of corn, and the nonpathogenic fungi Aspergillus nidulans and Neurospora crassa [14]. We then sought to identify unique Fusarium genes that may be critical for plant disease by identifying $F$. verticillioides ESTs that were most similar (BLASTX threshold values $\leq 10^{-200}$ ) to $F$. graminearum genome sequences and then subtracting all those ESTs sequences that had likely homologues in $A$. nidulans or $N$. crassa (BLASTX threshold values $\leq 10^{-5}$ ). Of the 39 Fusarium sequences identified via this process, five were similar to peptidases, three were similar to transcriptional or translational regulatory genes, 14 were similar primarily to genes 
Table 2. BLASTX analysis of sequences unique to pathogenic Fusarium

\begin{tabular}{lcccc}
\hline Putative function & Total $F^{\mathrm{a}}$ & $\mathrm{OF}^{\mathrm{b}}$ & $\mathrm{B}$ or $\mathrm{E}^{\mathrm{c}}$ & Only F $^{\mathrm{d}}$ \\
\hline Peptidases $\left(E \leq 10^{-45}\right)$ & 5 & 4 & 1 & 0 \\
Regulation $\left(E \leq 10^{-10}\right)$ & 3 & 3 & 0 & 0 \\
Other proteins $\left(E \leq 10^{-7}\right)$ & 14 & 4 & 10 & 0 \\
Unknown proteins & 17 & 8 & 1 & 8 \\
Total & 39 & 19 & 12 & 8 \\
\hline
\end{tabular}

${ }^{\mathrm{a}}$ Total $\mathrm{F}=$ Total number of sequences unique to Fusarium.

${ }^{\mathrm{b}} \mathrm{OF}=$ Other fungi including Magnaporthe grisea, A. fumigatus, A. niger, Cryptococcus neoformans, and Ustilago maydis.

${ }^{c}$ Bacterial or Eukaryote other than fungal.

${ }^{\mathrm{d}}$ Only F $=$ No similar sequence was found at NCBI.

involved in modifications of low molecular weight compounds or proteins (e.g. oxidase), and 17 were not similar to any previously described genes [14]. The expression profiles of all 39 genes in the eight different cDNA libraries used to generate the EST data do not suggest any particular trend that might support a role unique to a specific library. In general, transcripts for genes in each of the general categories depicted in Table 2 were present in a multiple libraries. For example, all five putative peptidases were represented in the $F$. verticillioides cDNA library derived from growth on excised maize seedlings tissue, only three were in the library derived from growth on corn meal medium, and only two were in libraries derived from growth in the liquid fumonisin production medium.

Interestingly, BLASTX analysis of the 39 genes against the NCBI database revealed that almost $50 \%$ shared significant homology $\left(E \leq 10^{-5}\right)$ to genes from other pathogenic fungi, including Aspergillus (Table 1), and $31 \%$ shared significant homology to genes from bacterial or eukaryotes other than fungi. Perhaps the homologous genes found in pathogenic Aspergillus were present in an evolutionarily distant fungal progenitor but were lost as $A$. nidulans evolved. At a minimum, this analysis indicates that one must be cautious when interpreting "unique gene sets" generated by filtering one gene set or genome against another. We are currently in the process of examining the potential role in disease of the three regulatory genes as well as the nine unknown genes unique to the two Fusarium species.

\section{The search for the "one": Mining genomic data}

The preliminary genomic sequence data from $F$. graminearum and $F$. verticillioides as well as other members of the Ascomycota, has allowed researchers to examine the evolution of large gene sets [20]. Analysis of portions of 14 PKSs from $F$. graminearum and 16 from $F$. verticillioides with the same region from other fungal PKSs provided evidence for numerous gene duplication and gene loss events leading to a significant diversity of fungal PKSs. Based on homology, only four sets of PKSs from these Fusarium appear to be orthologs $[20,21]$. From a functional standpoint, the most interesting result from this formal analysis was the support it provided for early observations that fungal PKSs could be divided into two major functional classes; the reducing class in which the PKS fully reduces keto groups that result from successive condensations of acetyl units to the growing polyketide and the non-reducing class in which the PKS typically does not fully reduce the keto group [20].

The greatest challenge facing researchers with genomic sequence data is deciding which gene, among the thousands identified, to functionally characterize. A typical $30-45 \mathrm{mb}$ filamentous fungal genome is estimated to encode approximately $12,000-15,000$ genes. Which of the estimated $200 \mathrm{Zn}(\mathrm{II})_{2} \mathrm{Cys}_{6}$ fungal type regulatory genes encoded by these genomes is important for disease? Which of the hundreds of cytochrome P450-, oxidase- and methyltransferase-encoding genes is involved in the biosynthesis of a metabolite of interest? One approach to narrow down the candidate gene pool for functional studies is to identify a subset of genes by similarity searches (e.g. all cytochrome P450 monooxygenases) and then disrupt the entire set. Integral to this approach is the assumption that at least one member of the targeted gene family affects the process of interest. A second approach is to examine gene expression profiles 
in a variety of conditions and identify those genes whose expression appear to be positively or negatively correlated to a particular process. The major advantage of the latter approach is that all genes identified are examined; there is no a priori bias to analyze genes with a known function.

One study in Fusarium utilizing the first approach targeted all PKS genes identified in the $F$. graminearum genomic sequence [22]. Polyketides contribute significantly to the broad diversity of fungal secondary metabolites of which many have useful pharmaceutical properties or are toxic to plants and animals. Of the 15 PKSs identified in $F$. graminearum, initial analysis of individual PKS mutants identified a function for five: two were responsible for the synthesis of the mycotoxin zearalenone, one for the synthesis of the mycotoxins fusarin $\mathrm{C}$ and one each for the synthesis of the red mycelial pigment aurofusarin and black perithecial pigment [22]. Additional research will be required to determine the function of the remaining PKSs.

\section{Microarrays}

Differential expression of messenger RNA has been used extensively to identify genes or gene products involved in specific biological processes. Two different strategies are being used to study the expression of many Fusarium genes in parallel with DNA microarrays. The first method focuses on a set of 716 genes expressed after growth on maize. The array was created by spotting PCR generated DNA directly onto glass slides. Initial analysis of these arrays found differential expression of numerous genes under different conditions [23]. The second strategy utilizes oligonucleotide-based arrays and a set of genes generated from either a large scale EST project (F. verticillioides; 11,126 genes) or from whole genome sequence (F. graminearum; $\sim 11,000$ genes). Each gene is represented on the array by up to 12 pairs of oligos, tiled across the gene, where one oligo is a perfect match and the other contains a single nucleotide difference [reviewed in 24]. Two companies, Affymetrix and NimbleGen, offer very

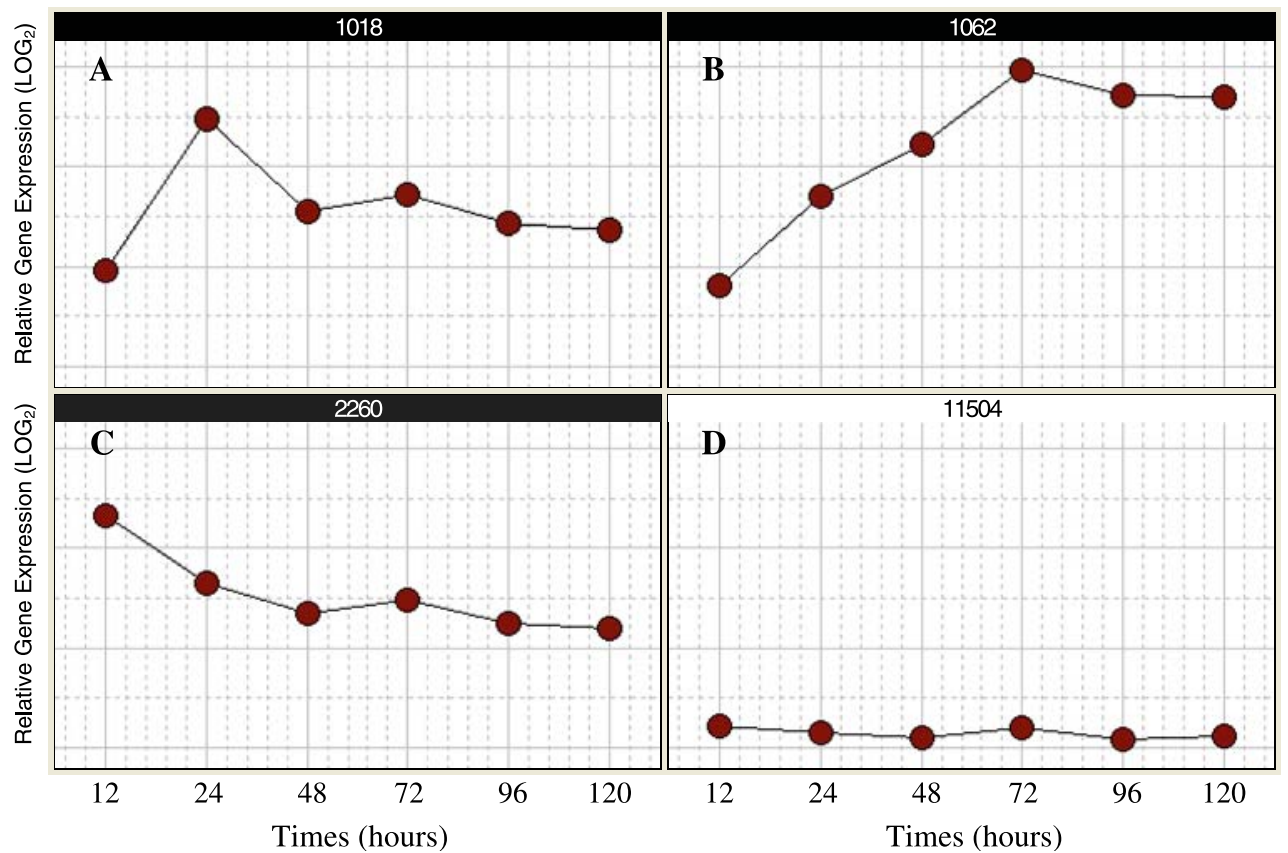

Figure 1. Microarray analysis of the expression of 15,844 Fusarium verticillioides gene sequences represented in the array over a period of 5 days. The data are presented as self organizing maps generated using Acuity 4.0 software (Molecular Devices). The four maps divide the expression of all 15844 gene sequences into four expression profiles. The number above each map represents the number of probe sets contributing to the expression pattern. Thus, maps A, B, C, and D represent the mean relative expression of 1018, 1062, 2260 and 11504 gene sequences, respectively. The data were generated with two biological reps. 
similar services where oligonucleotides are synthesized on slides at high-density by photolithography. Each slide, representing the entire gene call set, is then separately hybridized to labeled sample and expression levels are measured. A microarray designed by Affymetrix containing a F. graminearum gene set based on genomic sequence has been generated and is currently under evaluation (Frances Trail, Pers. Commun.). A microarray designed by NimbleGen, in collaboration with TIGR containing a $F$. verticillioides gene set based on EST sequence has been generated and is also currently under evaluation.

Preliminary experiments with the $F$. verticillioides oligo based microarray have provided intriguing results. One goal of initial experiments was to validate the microarray by hybridizing them with RNA extracted from mycelium cultured under fumonisin producing conditions; the same conditions used to generate four of the EST libraries that contributed to the $F$. verticillioides gene set [14]. Initial analysis of the array with RNA extracted over a five-day time course are interesting. As expected, FUM gene transcripts are minimal at $24 \mathrm{~h}$, increase markedly at $48 \mathrm{~h}$, and stay at high levels though $120 \mathrm{~h}$ (Figure 1). Over all, although the expression of most genes does not change $(11,504)$, significant numbers of genes were differentially expressed, including genes not previously found to be involved in fumonisin biosynthesis. For example, the expression of 1062 sequences increased and 2260 decreased markedly during the course of the experiment, while 1018 others peaked at $24 \mathrm{~h}$ (Figure 2). Future

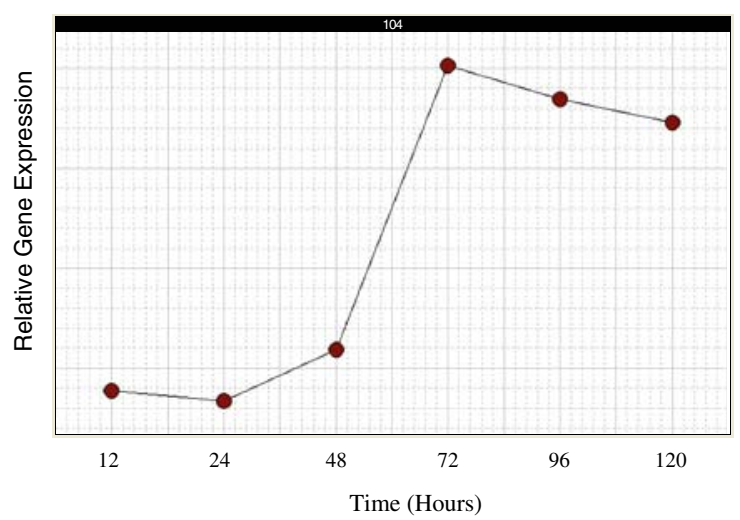

Figure 2. Relative gene expression profile of 104 individual sequences representing all $F U M$ cluster genes from a hybridization of RNA samples from six time points of wild-type Fusarium verticillioides cultured in liquid fumonisin production medium. experiments are aimed at using the arrays to identify new targets for investigation into regulation of secondary metabolism as well as pathogenicity.

\section{Conclusions}

Currently, the fungal genomics community is using microarrays to examine fungal gene transcription after growth in a variety of different conditions. This is just the beginning. In the next year or so, new genome sequence information coupled with better annotation technology will allow researchers to develop second generation arrays that more accurately represent the entire fungal repertoire of genes. Genomic resources have provided researchers with powerful tools to speed the process of identifying new fungal genes for further study. Understanding the role these genes play in mycotoxin biosynthesis and in pathogenesis during fungal-plant interactions will take time and resources. In 3-5 years, the field of proteomics will also impact our choice of genes for functional analysis. It is only by moving forward on numerous research fronts that we will meet our goal to develop new fungal control strategies and provide for a safer food supply for tomorrow.

\section{Note added in proof}

Work at MIPS has been described in Güldener U, Mannhaupt G, Münsterkötter M, Haase D, Oesterheld M, Stümpflen V, Mewes H, Adam G. FGDB: a comprehensive fungal genome resource on the plant pathogen Fusarium graminearum. Nucl Acids Res 2006; 34: D456-D458.

\section{Acknowledgements}

We thank Christopher McGovern, Crystal Platis, Debbie Shane and Marcie Moore for technical assistance.

\section{References}

1. Snyder WC. Introduction. In: Nelson PE, Toussoun TA, Cook RJ, eds. Fusarium: Diseases, Biology, and Taxonomy, The Pennsylvania State University PressUniversity Park, 1981: 1-8.

2. Burgess LW. General ecology of the Fusaria. In: Nelson PE, Toussoun TA, Cook RJ, eds. Fusarium: Diseases, 
Biology, and Taxonomy, The Pennsylvania State University PressUniversity Park, 1981: 225-235.

3. Bennett JW, Klich M. Mycotoxins. Clin Microbiol Rev 2003; 16: 497-516.

4. Brown DW, McCormick SP, Alexander NJ, Proctor RH, Desjardins AE. A genetic and biochemical approach to study trichothecene diversity in Fusarium sporotrichioides and Fusarium graminearum. Fungal Genet Biol 2001; 32: 121-144.

5. Gaffoor I, Trail F. Characterization of two distinct polyketide synthase genes involved in zearalenone biosynthesis in Gibberella zeae. Appl Environ Microbiol 2006; 72: $1793-$ 1799.

6. Proctor RH, Brown DW, Plattner RD, Desjardins AE. Co-expression of 15 contiguous genes delineates a fumonisin biosynthetic gene cluster in Gibberella moniliformis. Fungal Genet Biol 2003; 38: 237-249.

7. Butchko RA, Plattner RD, Proctor RH. FUM13 encodes a short chain dehydrogenase/reductase required for $\mathrm{C}-3$ carbonyl reduction during fumonisin biosynthesis in Gibberella moniliformi. J Agric Food Chem 2003; 51: 3000-3006.

8. Bennett JW. White paper: Genomics for filamentous fungi. Fungal Genet Biol 1997; 21: 3-7.

9. Jurgenson JE, Zeller KA, Leslie JF. Expanded genetic map of Gibberella moniliformis (Fusarium verticillioides). Appl Environ Microbiol 2002; 68: 1972-1979.

10. Trail F, Xu JR, San Miguel P, Halgren RG, Kistler HC. Analysis of expressed sequence tags from Gibberella zeae (anamorph Fusarium graminearum). Fungal Genet Biol 2003; 38: 187-197.

11. Jurgenson JE, Bowden RL, Zeller KA, Leslie JF, Alexander NJ, Plattner RD. A genetic map of Gibberella zeae (Fusarium graminearum). Genetics 2002; 160: 14511460.

12. O'Donnell K, Ward TJ, Geiser DM, Corby Kistler H, Aoki T. Genealogical concordance between the mating type locus and seven other nuclear genes supports formal recognition of nine phylogenetically distinct species within the Fusarium graminearum clade. Fungal Genet Biol 2004; 41: 600-623.

13. Xu JR, Leslie JF. A genetic map of Gibberella fujikuroi mating population A (Fusarium moniliforme). Genetics 1996; 143: 175-189.

14. Brown DW, Cheung F, Proctor RH, Butchko RAE, Zheng L, Lee Y, Utterback T, Smith S, Feldblyum T, Glenn AE, Plattner RD, Kendra DF, Town CD, Whitelaw CA. Comparative analysis of 87,000 expressed sequence tags from the fumonisin-producing fungus Fusarium verticillioides. Fungal Genet Biol 2005; 42: 848-861.

15. Flaherty JE, Woloshuk CP. Regulation of fumonisin biosynthesis in Fusarium verticillioides by a zinc binuclear cluster-type gene, ZFR1. Appl Environ Microbiol 2004; 70: 2653-2659.

16. Brown DW, Butchko RAE, Bussman M, Proctor RH. The fumonisin biosynthetic gene cluster in Fusarium verticillioides is regulated by a $\mathrm{Zn}(\mathrm{II}) 2 \mathrm{Cys} 6$ transcriptional factor. In preparation.

17. Kellis M, Patterson N, Endrizzi M, Birren B, Lander ES. Sequencing and comparison of yeast species to identify genes and regulatory elements. Nature 2003; 423: 241-254.

18. Nierman WC, May G, Kim HS, Anderson MJ, Chen D, Denning DW. What the Aspergillus genomes have told us. Med Mycol 2005; 43: S3-S5.

19. El-Sayed NM, Myler PJ, Blandin G, Berriman M, Crabtree J, Aggarwal G, Caler E, Renauld H, Worthey EA, Hertz-Fowler C, Ghedin E, Peacock C, Bartholomeu DC, Haas BJ, Tran AN, Wortman JR, Alsmark UC, Angiuoli S, Anupama A, Badger J, Bringaud F, Cadag E, Carlton JM, Cerqueira GC, Creasy T, Delcher AL, Djikeng A, Embley TM, Hauser C, Ivens AC, Kummerfeld SK, Pereira-Leal JB, Nilsson D, Peterson J, Salzberg SL, Shallom J, Silva JC, Sundaram J, Westenberger S, White O, Melville SE, Donelson JE, Andersson B, Stuart KD, Hall N. Comparative genomics of trypanosomatid parasitic protozoa. Science 2005; 309: 404-409.

20. Kroken S, Glass NL, Taylor JW, Yoder OC, Turgeon BG. Phylogenomic analysis of type I polyketide synthase genes in pathogenic and saprobic ascomycetes. Proc Natl Acad Sci USA 2003; 100: 15670-15675.

21. Callighan B, Johnson K, Brown DW. Isolation and sequence analysis of polyketide synthase genes from three Fusaria commonly found in corn. Fungal Genet News 2005; 52.

22. Gaffoor I, Brown DW, Plattner RD, Proctor RH, Qi W, Trail F. Functional analysis of the polyketide synthase genes in the filamentous fungus Gibberella zeae (anamorph Fusarium graminearum). Eukaryot Cell 2005; 4: 1926 1933.

23. Pirttila AM, McIntyre LM, Payne GA, Woloshuk CP. Expression profile analysis of wild-type and $f c c 1$ mutant strains of Fusarium verticillioides during fumonisin biosynthesis. Fungal Genet Biol 2004; 41: 647-656.

24. Aharoni A, Vorst O. DNA microarrays for functional plant genomics. Plant Mol Biol 2002; 48: 99-118.

Address for correspondence:Daren W. Brown, Mycotoxin Research GroupUSDA/ARS, 1815 N University St, Peoria, IL, 61604, USA

Phone: + 1-309-681-6230; Fax: + 1-309-681-6689

E-mail: browndw@ncaur.usda.gov 